\title{
VÖW-Sommerakademie 2016 „Industrial Ecology“ (10.-14.9.2016)
}

17. Mai 2016

(C) Springer-Verlag Berlin Heidelberg 2016

Industrial Ecology (IE) ist die Idee, Wirtschaften nach dem Vorbild von Ökosystemen zu gestalten: kreislauforientiert, ressourceneffizient, nachhaltig und zukunftsfähig. Die Sommerakademie der Vereinigung für ökologische Wirtschaftsforschung (VÖW) diskutiert Konzepte und Methoden der IE sowie ihre Bezüge zu anderen Feldern der Nachhaltigkeitsforschung. Sie findet vom 10.-14.9.2016 in Pforzheim statt und wird vom INEC der dortigen Hochschule ausgerichtet.
Die VÖW und das Netzwerk IE freuen sich auf die Teilnahme von Studierenden, AbsolventInnen und DoktorandInnen aller Fachrichtungen mit einem Grundwissen zu und Interesse an Fragen und Methoden der Nachhaltigkeit.

Weitere Informationen und Anmeldung:

www.voew.de/sommerakademie

Kontakt: sommerakademie@ voew.de 